\title{
Evaluation of the vertical vibrations behaviour of the bogie at failure of the dampers in the primary suspension of the railway vehicle
}

\author{
Mădălina Dumitriu* and Marius Alin Gheţi \\ University Politehnica of Bucharest, Department of Railway Vehicles, 313 Splaiul Independenţei, \\ Bucharest, Romania
}

\begin{abstract}
The paper aims to evaluate the behaviour of vertical vibrations of a two-axle bogie at failure of a damper in the primary suspension of the vehicle. The level of vibrations in the bogie is shown to rise as a consequence of the imbalance triggered in the system by the fault damper, resulting in dynamic interferences between the bounce and pitch vibrations of the bogie. This fact is highlighted by the results derived from the numerical simulations, which feature the frequency response functions of the bogie during rolling on a track with vertical irregularities. Various scenarios will be considered for lowering the damping constant in comparison with the reference value.
\end{abstract}

\section{Introduction}

While running, the rail vehicle undergoes a constant behaviour of vibrations, whose source is to be found in the phenomena of interaction between vehicle and track [1, 2]. In terms of level of vibrations, the suspension system plays a decisive role in regard to the capability of the vehicle to provide ride quality and running safety, comfort of the passengers and to keep the goods being transported in great condition [3, 4]. Similarly, the suspension assists with the reduction in the dynamic load to which both vehicle structure and track are subjected [5].

The suspension damages generally lead to decrease or increase of nominal parameters values - damping and stiffness coefficients [6]. Any deviation from the nominal characteristics of suspension influences the dynamic behaviour of the vehicle or, in extreme cases, it can jeopardize the security of passengers. Detecting component faults at their early stages prevents further deterioration in vehicle performance and enhances security while rapid and effective monitoring techniques are essential to increase vehicle reliability and reduce maintenance cost $[7,8]$.

The paper aims to evaluate the behaviour of vertical vibrations of a two-axle bogie in a particular case of a damper failure in the primary suspension of the railway vehicle. The behaviour of vibrations in the bogie is evaluated based on the response functions to the running on a track with vertical irregularities described by the power spectral density.

*Corresponding author: madalinadumitriu@yahoo.com 
Typically, in the studies from the speciality literature, the vertical bounce and pitch vibrations of the bogie are considered decoupled because of the use of elastic and damping components with identical characteristics in the primary suspension of each axle in a bogie and to the fact that the bogie is symmetrical from geometry and mass perspectives, compared to the vertical-transversal plan.

The results of the numerical simulations presented in the paper highlight the dynamic interferences between the bounce and pitch vibrations of the bogie at failure of a damper in the primary suspension of the bogie. Similarly, the level of vibrations in the bogie is shown to increase, due to the failure of the damper, where this expansion is closely linked to the failure degree, which is the damping decrease.

\section{The mechanical model of the bogie}

To evaluate the behaviour of vertical vibrations in a two-axle bogie at failure of a damper in the primary suspension of the vehicle, the mechanical model in Figure 1 is to be considered. The bogie travels at a constant speed on a track with vertical irregularities described corresponding to each axle by the functions $\eta_{1,2}$.

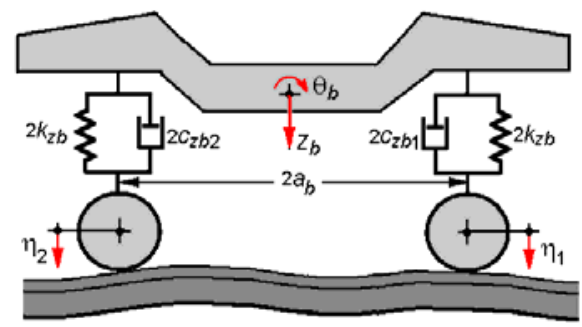

Fig. 1. The mechanical model of the bogie.

The modes of vibration of the bogie in a vertical plan - bounce $z_{b}$ and pitch $\theta_{b}$ (small angle) are being taken into account. The bogie parameters are follows: $m_{b}-$ mass of the bogie, $2 a_{b}$ - wheelbase, $J_{b}$ - moment of inertia. The primary suspension corresponding to each axle is modelled via Kelvin-Voigt system, with the elastic constant $2 k_{z b}$ and the damping constant $2 c_{z b 1}$ and $2 c_{z b 2}$, respectively. Applying the D'Alembert' principle, the bogie motion equations are as below:

$$
\begin{aligned}
& m_{b} \ddot{z}_{b}+\left(2 c_{z b 1}+2 c_{z b 2}\right) \dot{z}_{b}+4 k_{z b} z_{b}+\left(2 c_{z b 1}-2 c_{z b 2}\right) a_{b} \dot{\theta}_{b}= \\
&= 2 c_{z b 1} \dot{\eta}_{1}+2 c_{z b 2} \dot{\eta}_{2}+2 k_{z b}\left(\eta_{1}+\eta_{2}\right) \\
& J_{b} \ddot{\theta}_{b}+\left(2 c_{z b 1}+2 c_{z b 2}\right) a_{b}^{2} \dot{\theta}_{b}+4 k_{z b} a_{b}^{2} \theta_{b}+\left(2 c_{z b 1}-2 c_{z b 2}\right) a_{b} \dot{z}_{b}= \\
&=2 c_{z b 1} a_{b} \dot{\eta}_{1}-2 c_{z b 2} a_{b} \dot{\eta}_{2}+2 k_{z b}\left(\eta_{1}-\eta_{2}\right) .
\end{aligned}
$$

They feature the interactions between the bounce and pitch vibrations of the bogie at failure of the damper in the suspension of one of the two axles. As a rule, when neither of the dampers is damaged, the damping constants are equal $\left(2 c_{z b 1}=2 c_{z b 2}=2 c_{z b}\right)$; herein, the bogie motion equations are decoupled:

$$
\begin{gathered}
m_{b} \ddot{z}_{b}+4 c_{z b} \dot{z}_{b}+4 k_{z b} z_{b}=2 c_{z b}\left(\dot{\eta}_{1}+\dot{\eta}_{2}\right)+2 k_{z b}\left(\eta_{1}+\eta_{2}\right) ; \\
J_{b} \ddot{\theta}_{b}+4 c_{z b} a_{b}^{2} \dot{\theta}_{b}+4 k_{z b} a_{b}^{2} \theta_{b}=2 c_{z b} a_{b}\left(\dot{\eta}_{1}-\dot{\eta}_{2}\right)+2 k_{z b} a_{b}\left(\eta_{1}-\eta_{2}\right) .
\end{gathered}
$$




\section{The frequency response functions of the bogie}

It is considered that vertical irregularities of the track are in a harmonic shape with the wavelength $\Lambda$ and amplitude $\eta_{0}$.

$$
\eta_{1,2}(x)=\eta_{0} \cos (2 \pi / \Lambda)\left(x \pm a_{b}\right) \text { or } \eta_{1,2}(t)=\eta_{0} \cos \omega\left(t \pm a_{b} / V\right)
$$

Where $x=V t$ is the coordinate of the bogie centre, and $\omega=2 \pi V / \Lambda$ means the angular frequency induced by the track excitation.

Should the response of the bogie be harmonic with the same frequency as the frequency induced by the track excitation, the coordinates describing the movements of the bogie can be written as

$$
p_{k}(t)=P_{k} \cos \left(\omega t+\varphi_{k}\right)
$$

Where $P_{k}$ is the amplitude of the displacement and $\varphi_{k}$ - the phase shift of the coordinate $k$ compared with the track vertical irregularities at the centre of the bogie, with $k=1,2$.

When the complex units associated with real ones are introduced in the equations (1) and (2)

$$
\bar{\eta}_{i}(t)=\bar{\eta}_{i} e^{i \omega t} ; \bar{p}_{k}(t)=\bar{P}_{k} e^{i \omega t},
$$

a linear system of two non-homogeneous algebraic equations is obtained, whose solution allows the calculation of the frequency response functions of the bogie. The response function of the bogie acceleration is thus derived from the below relations

- at the bogie centre, $\quad \bar{H}_{a b}(\omega)=\omega^{2} \bar{H}_{z_{b}}(\omega)$,

- above the axles, $\quad \bar{H}_{a b 1,2}(\omega)=\omega^{2}\left[\bar{H}_{z_{b}}(\omega) \pm a_{b} \bar{H}_{\theta_{b}}(\omega)\right]$

Further on, the track vertical irregularities are represented by stochastic stationary process that can be defined via the power spectral density from the equation [9]

$$
S(\Omega)=\frac{A \Omega_{c}^{2}}{\left(\Omega^{2}+\Omega_{r}^{2}\right)\left(\Omega^{2}+\Omega_{c}^{2}\right)},
$$

where $\Omega$ is the wavelength, $\Omega_{c}=0.8246 \mathrm{rad} / \mathrm{m}, \Omega_{r}=0.0206 \mathrm{rad} / \mathrm{m} ; A=4.032 \cdot 10^{-7} \mathrm{radm}-$ for a for a high-level of track quality; $A=1.080 \cdot 10^{-6} \mathrm{radm}$ - for a low level of track quality.

As a function of the angular frequency $\omega=V \Omega$, the power spectral density of the track irregularities can be written as in the general relation

$$
G(\omega)=S(\omega / V) / V
$$

The equations (10) and (11) will give the power spectral density of the track vertical irregularities in the below form:

$$
G(\omega)=\frac{A \Omega_{c}^{2} V^{3}}{\left[\omega^{2}+\left(V \Omega_{c}\right)^{2}\right]\left[\omega^{2}+\left(V \Omega_{r}\right)^{2}\right]} .
$$


Starting from the response functions of the vehicle carbody eqs. (8) - (9) and the spectrum of the track irregularities eq. (12), the power spectral density of the bogie vertical acceleration is calculated:

- at the bogie centre,

$$
G_{a b}(\omega)=G(\omega)\left|\bar{H}_{a b}(\omega)\right|^{2}=G(\omega)\left|\omega^{2} \bar{H}_{z_{b}}(\omega)\right|
$$

- above the axles,

$$
G_{a b 1,2}(\omega)=G(\omega)\left|\bar{H}_{a b 1,2}(\omega)\right|^{2}=G(\omega) \mid \omega^{2}\left[\bar{H}_{z_{b}}(\omega) \pm\left. a_{b} \bar{H}_{\theta_{b}}(\omega)\right|^{2}\right.
$$

\section{Behaviour evaluation of the vertical vibrations in the bogie}

Based on the results from the numerical simulations, this section explores the behaviour of vibrations in the bogie at failure of a damper in the primary suspension of one of the two axles. The track vertical irregularities are described by the power spectral density (eq. 12) for a better-quality track $\left(A=4.032 \cdot 10^{-7} \mathrm{radm}\right)$. The parameters of the bogie used in the numerical simulations are included in Table 1.

In order to focus on the dynamic interferences between the bounce and pitch bogie vibrations at failure of one of the dampers, the phase shift of the track vertical irregularities at the two axles is not considered; therefore, it is considered $\eta_{1}(t)=\eta_{2}(t)=\eta_{0} \cos \omega t$.

Table 1. Parameters of numerical simulation.

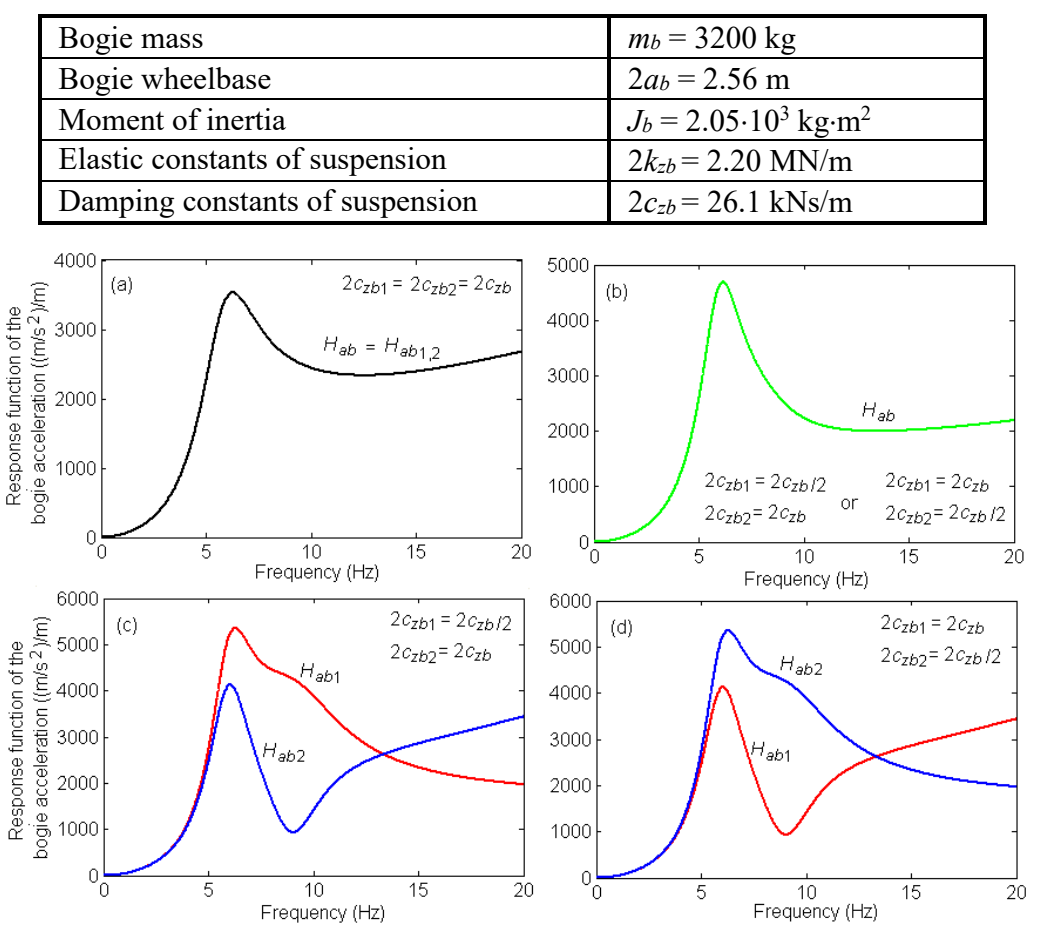

Fig. 2. The response function of the bogie acceleration. 
Under such conditions, as from equation (4), the pitch of the bogie is not excited if the dampers have equal damping constants. The bogie will, therefore, have only bounce movement both at its centre and above the axles. The same thing shows in Figure 2, (a), with the response function of the bogie acceleration at the velocity of $200 \mathrm{~km} / \mathrm{h}$.

When the damping constant is lowered in either suspension, the bogie pitch is excited and visible in the bogie response above the two axles, which is no longer symmetrical ((see. Figure 2, (c) and (d)). The level in the bogie vibrations rises (Figure 2, (b) - (d)), both due to the pitch excitation and the damping reduction, mainly at the resonance frequencies of the bounce and pitch vibrations $(5.9 \mathrm{~Hz}$ and $9.4 \mathrm{~Hz}$, respectively).

Figure 3 features the power spectral density of the bogie vertical acceleration calculated at the velocity of $200 \mathrm{~km} / \mathrm{h}$ against the suspension corresponding to each of the two axles. It should be mentioned that the phase shift of the track vertical irregularities at the two axles given by the bogie wheelbase will be taken into account. Firstly, the level of vibrations above the two axles is noticed to be different, due to the suspension damping and to the geometric filtering effect given by the wheelbase of the bogie [10]. Secondly, the reduction of the damping constant in either of the suspensions leads to the amplification of the response of the bogie above both axles.

For instance, the power spectral density of the acceleration rises from $0.099\left(\mathrm{~m} / \mathrm{s}^{2}\right)^{2} /(1 / \mathrm{s})$ - diagram (a) to $0.138\left(\mathrm{~m} / \mathrm{s}^{2}\right)^{2} /(1 / \mathrm{s})$ - diagram (b), or to $0.157\left(\mathrm{~m} / \mathrm{s}^{2}\right)^{2} /(1 / \mathrm{s})$ - diagram (c), above the rear bogie, at the frequency of $9.4 \mathrm{~Hz}$.
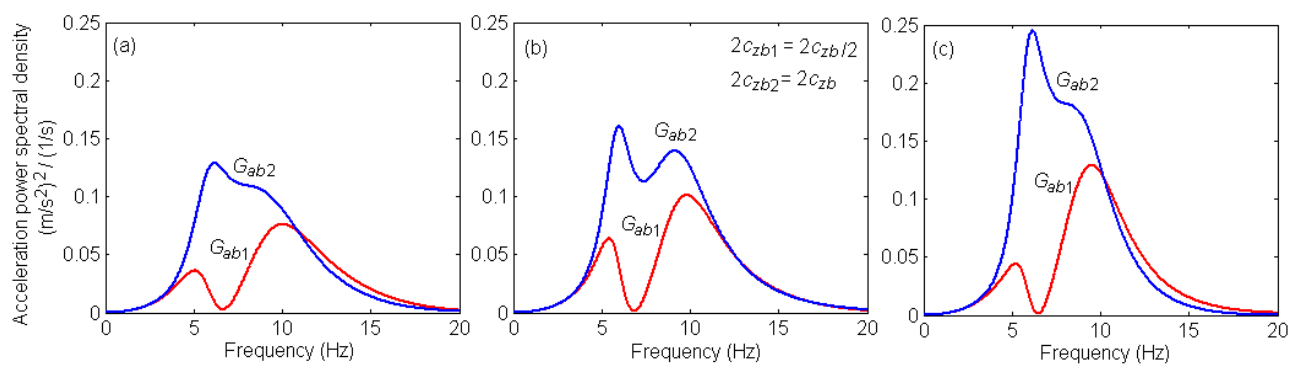

Fig. 3. The power spectral density of the bogie vertical acceleration for reducing the damping constant of the suspension to $1 / 2$ of the reference value.
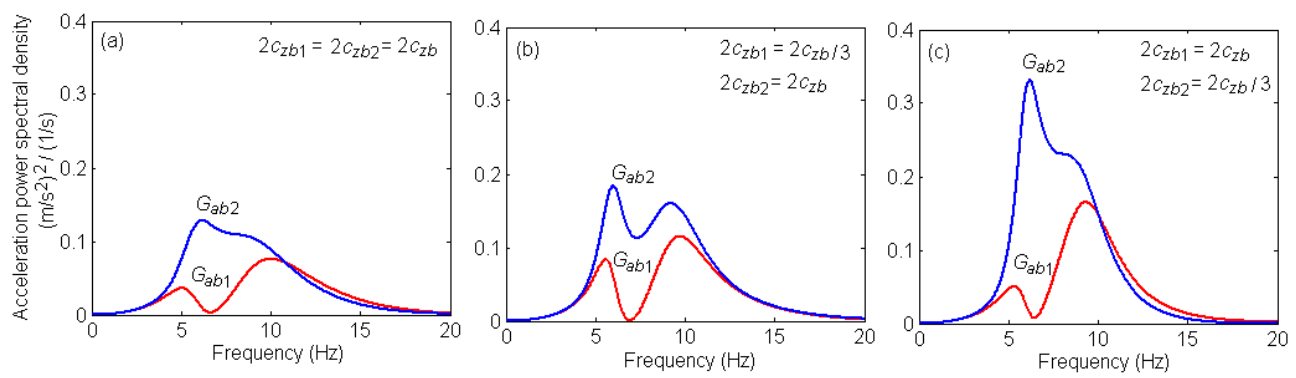

Fig. 4. The power spectral density of the bogie vertical acceleration for reducing the damping constant of the suspension to $1 / 3$ of the reference value.

It is a fact that the level of vibrations in the bogie goes up in dependence on the failure degree of the damper. This is clarified in the diagrams in Figure 4 and Figure 5, showing the power spectral density of the bogie acceleration at the velocity of $200 \mathrm{~km} / \mathrm{h}$, thus considering a reduction in the suspension damping constants to $1 / 3$ of the reference value and to $1 / 4$, respectively. 


\section{Conclusions}
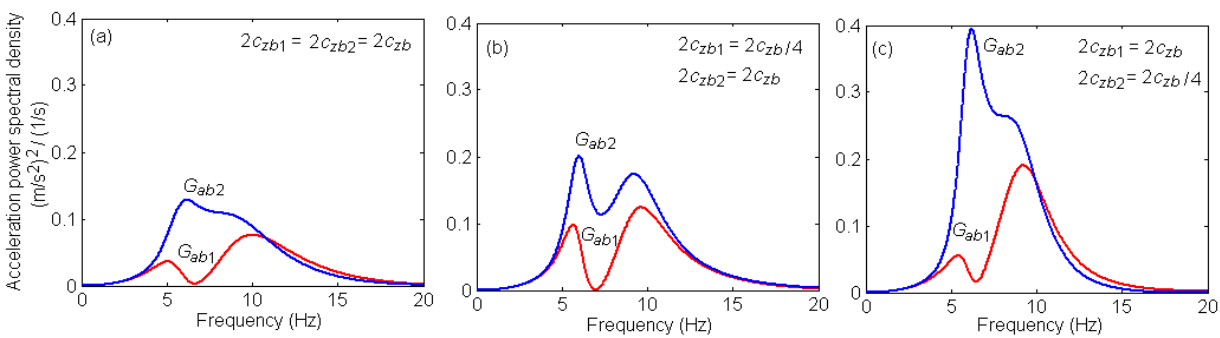

Fig. 5. The power spectral density of the bogie vertical acceleration for reducing the damping constant of the suspension to $1 / 4$ of the reference value.

The reliability of the suspension system in the railway vehicles is essential for meeting the criteria concerning the ride quality and ride comfort on the one hand and, on the other hand, for compliance with the conditions imposed by the traffic security. Any change in the suspension parameters due to a failure impacts the dynamic behaviour of the vehicle. The paper has examined the case of a damper failure in the primary suspension of a two-axle bogie from the perspective of the behaviour of vertical vibrations in the bogie. Dampers with identical characteristics are normally used in the suspension corresponding to each axle in the bogie, but this case will see the bounce and pitch vibrations decoupled. Based on the results from the numerical simulations, a failure in one of the dampers will mean a lower damping constant; dynamic interferences will occur between the two vibrations, triggering an amplification in the behaviour of the vertical vibrations in a bogie. The increase of the level of vibrations in the bogie against the suspension of each axle has been pointed out at for various situations of reducing the damping constant, compared with the reference value. The paper demonstrates that the change in the dynamic behaviour of the bogie as a result of the dynamic interference between the bounce and the pitch vibrations represents a consistent indication of the state of the primary suspension of the railway vehicle and this fact is sufficient to develop methods of fault detection in the dampers.

This work was supported by a grant of the Romanian National Authority for Science Research and Innovation, CNCS/CCDI - UEFISCDI, project number PN-III-P2-2.1-PED-2016-0212, within PNCDI III.

\section{References}

1. T. Mazilu, Proceedings of the Romanian Academy, Series A: Mathematics, Physics, Technical Sciences, Information Science, 10, 3 (2009)

2. T. Mazilu, Proceedings of the Romanian Academy, Series A: Mathematics, Physics, Technical Sciences, Information Science, 10, 2 (2009)

3. M. Dumitriu, Archive of Mechanical Engineering, LXI, 4 (2014)

4. M. Dumitriu, UPB Scientific Bulletin, Series D: Mechanical Engineering, 74, 4 (2012)

5. M. Dumitriu, UPB Scientific Bulletin, Series D: Mechanical Engineering, 75, 1 (2013)

6. R. Melnik, B. Sowiński, 7th Europ. Workshop on Structural Health Monitoring, (2014)

7. S. Bruni, R. Goodall, T.X. Mei, et al. Vehicle System Dynamics, 45, 7-8 (2007)

8. P. Li, R. Goodall, P. Weston, C.S. Ling, et al., Control Engineering Practice, 15 (2007)

9. ORE C 116/RP1, Interaction between vehicles and track (Utrecht, 1971)

10. M. Dumitriu, Journal of Engineering Science and Technology Review, 8, 4 (2015) 